Tropical Journal of Pharmaceutical Research March 2017; 16 (3): 613-620

ISSN: $1596-5996$ (print); 1596-9827 (electronic)

(c) Pharmacotherapy Group, Faculty of Pharmacy, University of Benin, Benin City, 300001 Nigeria.

All rights reserved.

Available online at http://www.tjpr.org

Original Research Article

http://dx.doi.org/10.4314/tjpr.v16i3.16

\title{
Antibacterial and anti-breast cancer cell line activities of Sanghuangporus sp.1 extracts
}

\author{
Kusavadee Sangdee', Benjaporn Buranrat', Khwanyuruan Naksuwankul ${ }^{1}$, \\ Piyanoot Jaihan ${ }^{2}$ and Aphidech Sangdee ${ }^{2,3 *}$ \\ ${ }^{1}$ Biomedical Sciences Research Unit, Faculty of Medicine, Mahasarakham University, Muang District, Maha Sarakham 44000, \\ ${ }^{2}$ Department of Biology, Faculty of Science, ${ }^{3}$ Microbiology and Applied Microbiology Research Unit, Faculty of Science, \\ Mahasarakham University, Kantarawichai District, Maha Sarakham 44150, Thailand
}

*For correspondence: Email: aphidech.s@msu.ac.th, aphidech_sangdee@yahoo.com; Tel: 6681-2615464; Fax: 6643-754245

Received: 7 December 2016

Revised accepted: 19 February 2017

\begin{abstract}
Purpose: To evaluate the activity of extracts of Sanghuangporus sp. 1 fungus against pathogenic bacteria and a breast cancer cell line.

Methods: The wild fruiting body and mycelium of Sanghuangporus sp. 1 were extracted with water and ethanol by ultrasonication extraction. The activity of the extracts against pathogenic bacteria was determined by agar-well diffusion method while minimum inhibitory concentration (MIC) and minimum bactericidal concentration (MBC) were obtained by agar-well diffusion and broth macrodilution methods. The cytotoxicity of the extract against human breast cancer cell line MCF-7 was determined by sulforhodamine $B$ assay.

Results: Only the ethanol mycelial extract exhibited antibacterial activity. Activity was detected against 6 of the 17 test strains of bacterial pathogen. The MICs and MBCs against these 6 strains were quite low, especially for B. cereus ATCC 11778 (2.5 and $2.5 \mathrm{mg} / \mathrm{mL}$ ) and S. aureus (MSSA, DMST 2933, 2.5 and $5.0 \mathrm{mg} / \mathrm{mL}$ ). The ethanol mycelial extract was a more potently cytotoxic against MCF-7 cells than either the aqueous mycelial extract or the ethanol wild fruiting body extract, inhibiting cell growth at a concentration of $250 \mu \mathrm{g} / \mathrm{mL}$. The aqueous wild fruiting body extract was inactive against MCF-7 cells when compared with untreated control groups.

Conclusion: The ethanol mycelial extract of the medicinal mushroom Sanghuangporus sp.1, obtained by ultrasonication extraction method, exhibited potent antibacterial and anticancer activity and seems to be a substitute for wild Sanghuangporus $s p$.
\end{abstract}

Keywords: Antibacterial activity, Anticancer activity, Sanghuangporus sp.1, Mycelial

Tropical Journal of Pharmaceutical Research is indexed by Science Citation Index (SciSearch), Scopus, International Pharmaceutical Abstract, Chemical Abstracts, Embase, Index Copernicus, EBSCO, African Index Medicus, JournalSeek, Journal Citation Reports/Science Edition, Directory of Open Access Journals (DOAJ), African Journal Online, Bioline International, Open-J-Gate and Pharmacy Abstracts

\section{INTRODUCTION}

Mushrooms have been used as traditional remedies for many years [1], playing an important role in traditional medicine in Japan, China, Korea and other Asian countries [2]. The bioactivity of medicinal mushrooms is due to constituents such as polysaccharides, glycoproteins, proteoglycans, fatty acids and proteins [3]. Macrofungi in the genus Phellinus, especially Phellinus linteus, are reported to have many potentially salutary effects including immunomodulatory, anti-tumor, anti-oxidant, antiinflammatory and antimicrobial activities [4-10]. For example, acidic polysaccharides isolated from $P$. linteus induce the functional maturation of dendritic cells [11], and inotilone has in vitro and in vivo anti-inflammatory activities that might be related to increased catalase, superoxide dismutase and glutathione peroxidase activity [7]. 
Hur et al [4] found that methanol extract from $P$. linteus inhibits the growth of methicillin-resistant Staphylococcus aureus (MRSA).

Another medicinal mushroom in this genus is $P$. igniarius. Song et al [12] have demonstrated that $P$. igniarius extracts inhibit the proliferation of human hepatocarcinoma SK-Hep-1 cell lines in a dose-dependent manner. A polyphenol extract from $P$. igniarius has been reported to protect against acrolein toxicity in vitro, and against stroke in a mouse model [13]. Chang et al. [14] and Ayala-Zavala et al. [6] found that extracts from $P$. merrillii, $P$. gilvus, $P$. rimosus and $P$. badius have antioxidant activity. Moreover, the extracts from $P$. gilvus, $P$. rimosus and $P$. badius could inhibit growth of the fungus Alternaria alternata [6]. Extracts from $P$. baumii have activity against influenza and methicillin-resistant $S$. aureus $[15,16]$. These findings indicate that many fungi in the genus Phellinus produce bioactive compounds, and represent a potential source of new therapeutic agents. Some of the above species have recently been reclassified, including $P$. linteus, which has been moved from the Phellinus genus to the Sanghuangporus genus, and is now known as Sanghuangporus linteus.

Many macrofungi in the genera Phellinus and Sanghuangporus have been found in the northeast of Thailand, but limited information is available on their bioactive compounds. Only 3 species, $P$. igniarius, $P$. gilvus and $P$. merrillii, have been reported to have antibacterial activity. Activity was detected against the pathogenic bacteria Escherichia coli, Pseudomonas aeruginosa and $S$. aureus, [17-19]. Other Sanghuangporus and Phellinus species merit investigation as source of new products that could be developed as novel therapeutic agents in the future. Therefore, the aim of the present study was to isolate the medicinal Sanghuangporus fungus from the wild fruiting body and then evaluate the activity of different extracts against Gram-negative and Grampositive pathogenic bacteria by the agar well and macro-dilution method. The effect of these extracts on a breast cancer cell line was also examined.

\section{EXPERIMENTAL}

\section{Fungal isolation}

The wild fruiting body of the medicinal mushroom used in this study was provided by the Natural Medicinal Mushroom Museum, Mahasarakham University, Thailand (J. Naksuwankul 720 (MSUT)) (Figure 1). The Sanghuangporus sp.1 fungus fruiting bodies were washed several times with sterile distilled water and then the inner tissue of the fruiting bodies was cut into small pieces $\left(5 \times 5 \mathrm{~mm}^{2}\right)$ before being surface sterilized and transferred to potato dextrose agar (PDA) containing $100 \mathrm{mg} / \mathrm{mL}$ ampicillin. After 7 days, the white mycelium growing out of the fungal tissue was sub-cultured on potato dextrose agar (PDA) and incubated at $25-28{ }^{\circ} \mathrm{C}$.

\section{Preparation of extracts}

Mycelial discs of the Sanghuangporus sp.1 fungus were cut with a sterile cork borer and placed into $25 \mathrm{~mL}$ of PDB medium. The culture was incubated statically at $28{ }^{\circ} \mathrm{C}$ for 20 days. The mycelium on the surface of the culture was collected and dried at $50^{\circ} \mathrm{C}$ for 2 - 3 days before being powdered using a mortar and pestle. Five hundred milligram aliquots of the dried mycelium were placed in conical screw cap tubes and dissolved in either $12.5 \mathrm{~mL}$ of distilled water or $50 \%$ ethanol. The mycelium suspension (40 $\mathrm{mg} / \mathrm{mL}$ ) was then sonicated using the ultrasonication extraction method described by Sangdee and Sangdee [20]. The dried wild fruiting body was cut into small pieces before being powdered using a mortar and pestle. Aqueous and ethanol extraction of the wild fruiting body was performed as described above.

\section{Test bacteria}

All the extracts were screened for activity against 15 reference strains of bacteria (Bacillus cereus ATCC 11778, Escherichia coli [EIEC] DMST 30545, E. coli O157:H7 DMST 12743, , Salmonella enterica serovar Typhimurium ATCC 14028, S. enterica serovar Typhi DMST 22842, S. enterica serovar Typhi DMST 16122, Shigella flexneri DMST 4423, Sh. flexneri DMST 17569, Sh. dysenteriae DMST 15110, Staphylococcus aureus [MSSA] DMST 2933, S. aureus [MRSA] DMST 4738, S. aureus [MRSA] DMST 20651, $S$. aureus [MRSA] DMST 20654, Pseudomonas aeruginosa ATCC 27853 and Vibrio cholerae [01] DMST 9700). The 12 DMST reference strains were obtained from the Culture Collection for Medical Microorganisms, Department of Medical Sciences, Thailand. Two clinical isolates were also tested (Klebsiella pneumoniae and Pseudomonas aeruginosa).

\section{Primary screening for antibacterial activity}

The antibacterial activity of the extracts was initially screened using an agar well diffusion method. The screening method was performed as described by Sangdee et al [21]. Briefly, the 15 test bacteria were cultured by inoculating a 
single bacterial colony into Mueller Hinton broth (MHB), and then incubating with shaking (150 rpm) at $37{ }^{\circ} \mathrm{C}$ for $3 \mathrm{~h}$. The cultures were then adjusted to the turbidity of a $0.5 \mathrm{McF}$ arland standard. These cell suspensions were inoculated and spread over the entire surface of each Mueller Hinton agar (MHA) plate, and the agar plates were then cut using a sterile cork borer. Finally, $0.1 \mathrm{~mL}$ of $40 \mathrm{mg} / \mathrm{mL}$ of the extracts was added to each well (4 mg per well). After incubation at $37^{\circ} \mathrm{C}$ for $16-18 \mathrm{~h}$, the zones of inhibition in each plate were measured. Ciprofloxacin at a concentration of $250 \mu \mathrm{g} / \mathrm{mL}$ was used as a reference standard.

\section{Minimum inhibitory concentration (MIC) and minimum bactericidal concentration (MBC) assays}

For extracts that showed a detectable zone of inhibition against the test bacteria, this activity was quantified by MIC and MBC assay. The MICs of the extracts were determined using the agar well diffusion method and broth macrodilution method. The agar well diffusion method was performed as described above, but testing a series of concentrations $(40,20,10,5$, 2.5 and $1.25 \mathrm{mg} / \mathrm{mL}$ ). The MIC represented the lowest concentration of extract capable of inhibiting bacterial growth around the well. The broth macrodilution method involved preparing a series of concentrations of extract $(40,20,10,5$, 2.5 and $1.25 \mathrm{mg} / \mathrm{mL}$ ) in MHB medium, then inoculating each with $0.1 \mathrm{~mL}$ of bacterial suspension. The final cell density of bacteria was $10^{5} \mathrm{CFU} / \mathrm{mL}$. The mixtures were incubated with shaking for $24 \mathrm{~h}$ (150 rpm). MICs were determined by identifying the solutions that were clear after incubation compared with the control tubes (MHB plus test bacteria, MHB plus the extracts, and MHB plus antibiotic and test bacteria). MBCs were determined by transferring bacterial suspensions from the MIC assay onto MHA plates. The lowest concentration that resulted in no growth after $24 \mathrm{~h}$ incubation was recorded as the MBC. Ciprofloxacin and tetracycline, each at a concentration of 250 $\mu \mathrm{g} / \mathrm{mL}$ were used as reference standards. Two strains of methicillin-resistant Staphylococcus aureus (MRSA), viz, S. aureus DMST 4738 and S. aureus DMST 20654 at final cell density of $10^{5}$ $\mathrm{CFU} / \mathrm{mL}$ were used as test bacteria in the study.

\section{Evaluation of cytotoxicity against human breast cancer cells}

The effects of the extracts on the human breast cancer cell line MCF-7 were determined as described by Buranrat et al [22] using the sulforhodamine B (SRB) assay. Briefly, MCF-7 cells were seeded into a 96-well plate $\left(1 \times 10^{4}\right.$ cells/well) for $24 \mathrm{~h}$ before being exposed to the extracts at various concentrations for $72 \mathrm{~h}$. After that, the cultured medium was removed, fixed with ice-cold methanol, stained with $0.4 \%$ SRB, and solubilized with $10 \mathrm{mM}$ Tris-base buffer. The absorbance was measured at $540 \mathrm{~nm}$ using a microplate reader compared with untreated control groups.

\section{RESULTS}

\section{Fungal isolation and colony morphology}

Within 7 - 10 days, the mycelium of the fungus Sanghuangporus sp. 1 grew out of the tissue of the wild fruiting body and formed white colonies on PDA. The mycelium was sub-cultured onto a new PDA plate for morphological examination. After 3 - 5 days, the color of the colonies was white, these then changing to white-cream cottony colonies by $10-14$ days. The colonies grew quickly, covering the surface of the $90 \mathrm{~mm}$ PDA plate by 21 days (Figure 1 ). This isolate was assigned the reference number Sanghuangporus sp.1.

\section{Antibacterial activity of mycelial and fruiting body extracts}

In this study, mycelial extracts and wild fruiting body extracts of Sanghuangporus sp.1 were tested against 15 strains of pathogenic bacteria using an agar well diffusion method. The results showed that only the ethanolic mycelial extract exhibited antibacterial activity. Activity was detected against 4 of the 15 test bacterial strains including $S$. aureus [MSSA] DMST 2933, S. aureus [MRSA] DMST 20651, B. cereus ATCC 11778 and Vibrio cholerae [O1] DMST 9700. The aqueous mycelial extract, aqueous wild fruiting body extract, and ethanolic wild fruiting body extract had no effect against the test bacteria. The inhibition zones of the ethanolic mycelial extract ranged from $11-24 \mathrm{~mm}$, whereas the inhibition zones of the antibiotic ciprofloxacin ranged from $15-34 \mathrm{~mm}$. Interestingly, 3 of the test bacteria, $B$. cereus ATCC 11778, S. aureus [MSSA] DMST 2933 and S. aureus [MRSA] DMST 20651, were more sensitive to the ethanolic extract than to ciprofloxacin. Moreover, S. aureus (MRSA) DMST 20651 was sensitive to the ethanolic mycelial extract, but resistant to ciprofloxacin (Table 1).

The MIC and MBC values of the ethanolic mycelial extract were determined against the sensitive strains above and against two strains of methicillin-resistant Staphylococcus aureus, strains DMST 4738 and DMST 20654. 


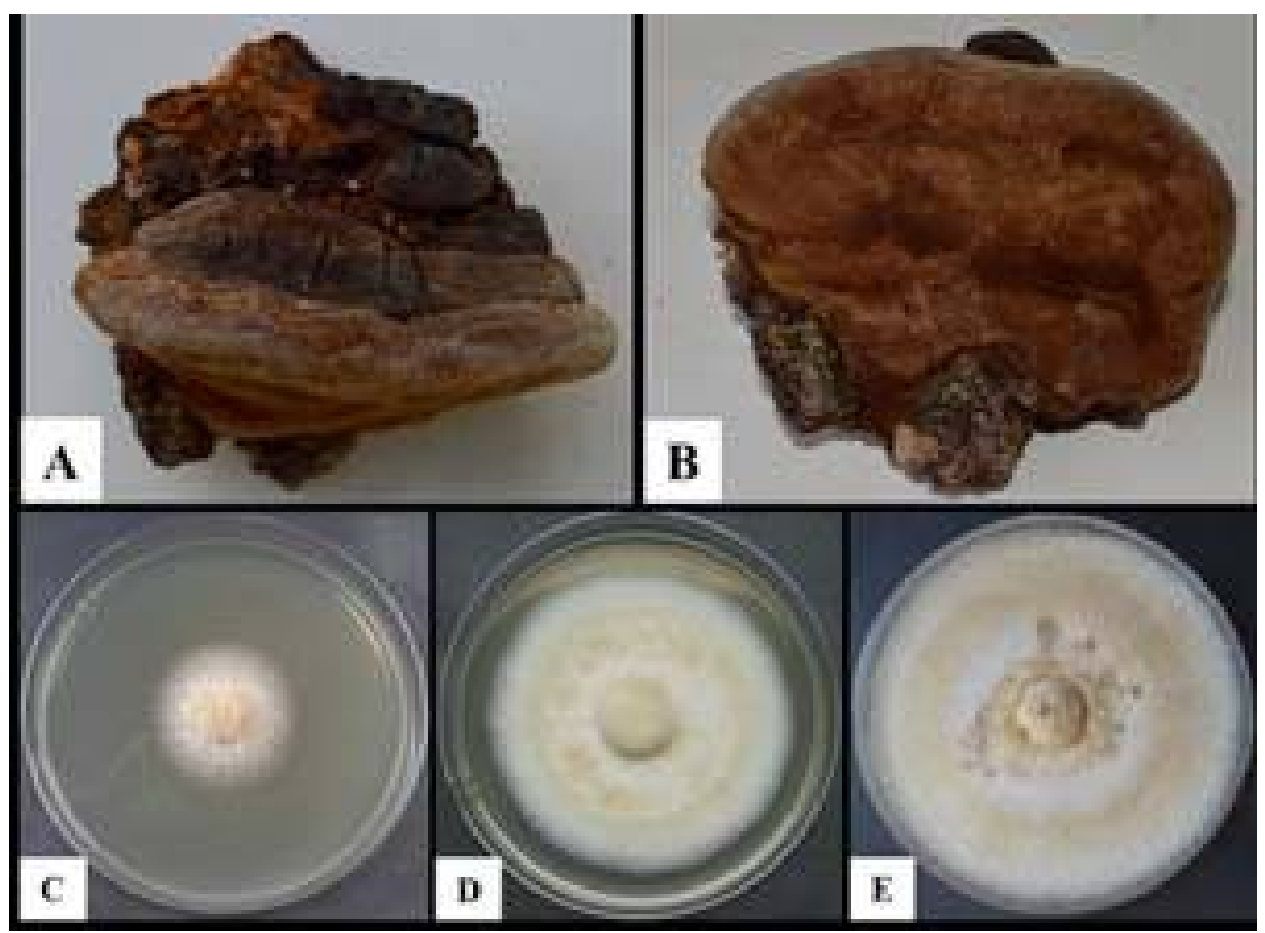

Figure 1: Basidiocarp (A and B) and a colony of the medicinal mushroom Sanghuangporus sp.1 on PDA medium at 7,14 and 22 days (C, D and E). (J. Naksuwankul 720 (MSUT)

Table 1: Primary antibacterial screening of the aqueous mycelial extract, ethanolic mycelial extract, aqueous fruiting body extract, and ethanolic fruiting body extract of Sanghuangporus sp.1 using the antibiotic ciprofloxacin as a reference standard

\begin{tabular}{lccccc}
\hline \multirow{2}{*}{ Test bacterium } & \multicolumn{5}{c}{ Zone of inhibition (mm) } \\
\cline { 2 - 5 } & \multicolumn{2}{c}{ Mycelial extract } & Fruiting body extract & Cipro (25 $\boldsymbol{\mu g})$ \\
\cline { 2 - 5 } B. cereus ATCC 11778 & 0 & EtOH & Aqueous & EtOH & \\
S. aureus (MSSA) DMST 2933 & 0 & $20 \times 23$ & 0 & 0 & $15 \times 15$ \\
S. aureus (MRSA) DMST 4738 & ND & ND & ND & ND & ND \\
S. aureus (MRSA) DMST 20651 & 0 & $24 \times 24$ & 0 & 0 & 0 \\
S. aureus (MRSA) DMST 20654 & ND & ND & ND & ND & ND \\
V. cholerae (O1) DMST 9700 & 0 & $11 \times 11$ & 0 & 0 & $34 \times 34$ \\
E. coli (EIEC) DMST 30545 & 0 & 0 & 0 & 0 & $13 \times 13$ \\
E. coli O157:H7 DMST 12743 & 0 & 0 & 0 & 0 & $25 \times 25$ \\
Sh. flexneri DMST 4423 & 0 & 0 & 0 & 0 & $26 \times 26$ \\
Sh. flexneri DMST 17569 & 0 & 0 & 0 & 0 & $29 \times 29$ \\
S. Typhi DMST 22842 & 0 & 0 & 0 & 0 & $30 \times 30$ \\
S. Typhi DMST 16122 & 0 & 0 & 0 & 0 & $25 \times 25$ \\
Sh. dysenteriae DMST 15110 & 0 & 0 & 0 & 0 & $30 \times 30$ \\
S. Typhimurium ATCC 14028 & 0 & 0 & 0 & 0 & $26 \times 26$ \\
Ps. aeruginosa ATCC 27853 & 0 & 0 & 0 & 0 & $27 \times 27$ \\
Ps. aeruginosa & 0 & 0 & 0 & 0 & $18 \times 18$ \\
K. pneumonia & 0 & 0 & 0 & 0 & $23 \times 23$ \\
\hline
\end{tabular}

EtOH, ethanol; Cipro, ciprofloxacin; ND, not determined; MIC, minimum inhibitory concentration; MBC, minimum bactericidal concentration

The MICs of the ethanolic mycelial extract were quite low ranging from $2.5-10 \mathrm{mg} / \mathrm{mL}$ by the agar well diffusion method. The MIC values of the same extract measured by the broth macrodilution method were $2.5-5 \mathrm{mg} / \mathrm{mL}$. B. cereus ATCC 11778 and S. aureus [MSSA] DMST 2933 were the most sensitive. The MBC values of the ethanolic mycelial extract ranged from $2.5-5.0 \mathrm{mg} / \mathrm{mL}$, with $B$. cereus ATCC 11778 being the most sensitive (Table 2). 
Table 2: MIC and MBC values of the ethanolic mycelial extract of Sanghuangporus sp.1 against 6 strains of pathogenic bacteria

\begin{tabular}{lccc}
\hline Test bacterium & \multicolumn{2}{c}{ MIC $(\mathbf{m g} / \mathbf{m L})$} & MBC $(\mathbf{m g} / \mathbf{m L})$ \\
\cline { 2 - 3 } & Agar well method & $\begin{array}{c}\text { Broth macrodilution } \\
\text { method }\end{array}$ & \\
\hline B. cereus ATCC 11778 & 2.5 & 2.5 & 2.5 \\
S. aureus (MSSA) DMST 2933 & 2.5 & 2.5 & 5.0 \\
S. aureus (MRSA) DMST 20651 & 10.0 & 5.0 & 5.0 \\
S. aureus (MRSA) DMST 4738 & 5.0 & 5.0 & 5.0 \\
S. aureus (MRSA) DMST 20654 & 10.0 & 5.0 & 5.0 \\
V. cholerae (O1) DMST 9700 & 10.0 & 5.0 & 5.0 \\
\hline
\end{tabular}

\section{Cytotoxicity against human breast cancer cells}

The cytotoxicity of the extracts against MCF-7 cells was evaluated using the SRB assay. The results showed that MCF-7 cells were more sensitive to the mycelial extracts than the wild fruiting body extracts. The ethanolic mycelial extract had greater cytotoxicity against the MCF7 cells than either the aqueous mycelial extract or the ethanolic wild fruiting body extract. No activity was detected from the aqueous wild
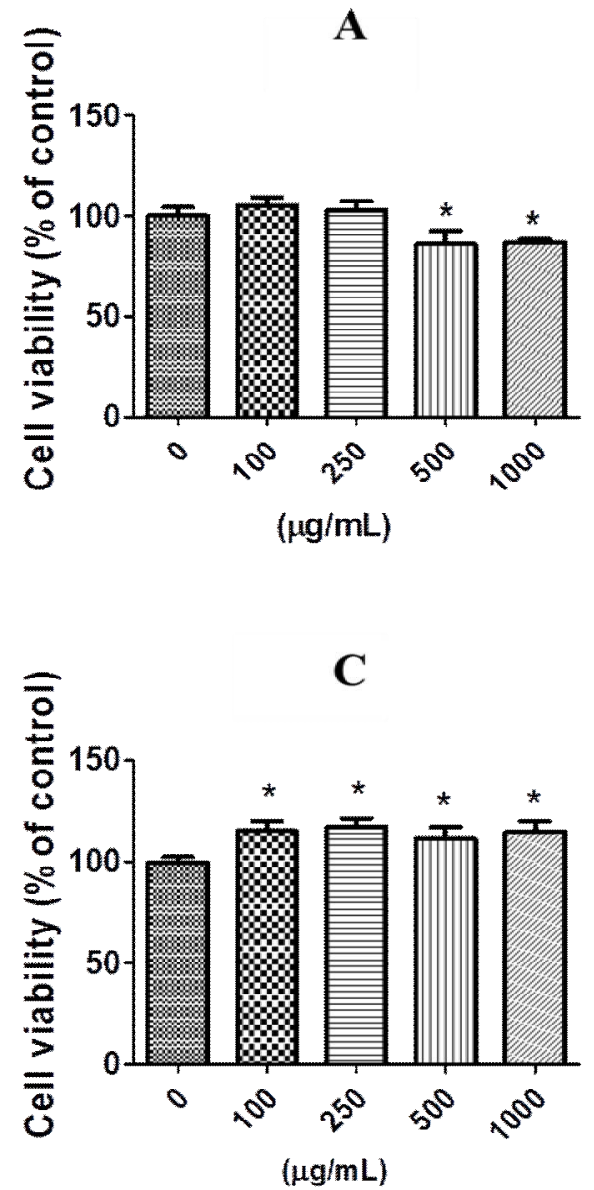

fruiting body extract against the MCF-7 cells. At $72 \mathrm{~h}$, the aqueous and ethanolic mycelial extracts at concentrations of $500 \mu \mathrm{g} / \mathrm{mL}$ and 250 $\mu \mathrm{g} / \mathrm{mL}$ inhibited MCF-7 cell growth when compared to untreated cells (Figure 2). Moreover, the results showed that cell growth was strongly inhibited by ethanolic mycelial extracts in a dose-dependent manner with an $\mathrm{IC}_{50}$ value of $1122.67 \pm 203.09 \mu \mathrm{g} / \mathrm{mL}$, while no activity was detected from the aqueous wild fruiting body extract against the MCF-7 cells.
B
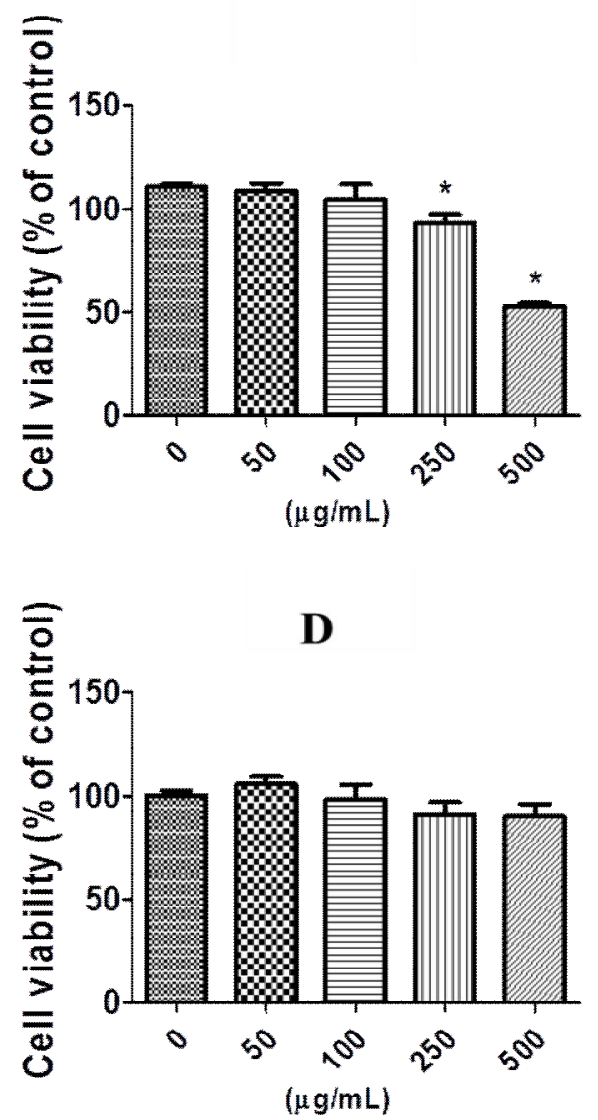

Figure 2: Effect of the aqueous mycelial extract (A), ethanol mycelial extract (B), aqueous wild fruiting body extract (C) and ethanol fruiting body extract (D) of Sanghuangporus sp.1 on the growth of MCF-7 cells. The cells were cultured for $24 \mathrm{~h}$ in 96-well plates and then treated with various concentrations of the extracts for $72 \mathrm{~h}$ before cell viability was determined by the SRB assay. The bars show mean \pm SEM, each from three independent experiments; ${ }^{*} p<0.05$ vs. untreated control groups 


\section{DISCUSSION}

In recent years, it has been proposed that several mushrooms belonging to the genera Sanghuangporus, Phellinus and Inonotus, such as S. linteus, Inonotus obliquus, I. xeranticus and $P$. ignarius could be used as nutritionally functional foods or as sources of medicinally useful compounds [23]. Examples of bioactive constituents include polysaccharides, especially $\beta$-glucan, which enhances immune system activity [24]. They also produce compounds with antioxidant activity $[25,26]$. In the present study, Sanghuangporus sp.1 was isolated and screened for antibacterial and anticancer activities.

The activity of artificially cultivated Sanghuangporus sp.1 extracts were compared with those of the wild fruiting body. The results indicate that the ethanolic mycelial extract has superior activity against $S$. aureus [MSSA] DMST 2933, S. aureus [MRSA] DMST 20651, S. aureus [MRSA] DMST 4738, $S$. aureus [MRSA] DMST 20654, B. cereus ATCC 11778 and Vibrio cholerae [01] DMST 9700 than extracts from the wild fruiting body. Interestingly, the ethanolic mycelial extract had activity against the medically important pathogenic bacteria S. aureus [MSSA] DMST 2933, S. aureus (MRSA) DMST 20651 and S. aureus (MRSA) DMST 20654, the latter 2 strains being resistant to ciprofloxacin. These results correlate well with those of Hur et al [4], who demonstrated that extracts from $S$. linteus have good activity against methicillin-resistant Staphylococcus aureus.

Similar findings to those above were observed in the cytotoxicity assay, with both mycelial extracts having greater cytotoxicity against the MCF-7 breast cancer cell line than the wild fruiting body extracts. These results indicate that mycelia cultivated in the laboratory are more bioactive, and may produce more potent medicinal compounds. These results correlate well with those of Sun et al [27], who demonstrated that artificially cultivated $l$. obliquus have similar inhibitory activity against tumor cells and bioactive compounds as wild I. obliquus. Our findings also suggest that the bioactivity of the ethanolic mycelial extract of Sanghuangporus sp.1 is significantly greater than the other extracts.

This comparative study indicates that the medicinal mushroom Sanghuangporus sp. 1 can be grown in the laboratory and used as a source of broad-spectrum antibacterial and anticancer agents. It can therefore be considered an alternative to wild Sanghuangporus spp.
However, further studies will be required to isolate and identify the compounds responsible for the anticancer properties of the extracts and elucidate the mechanism of action of ethanolic mycelial extract against breast and other cancer cells.

Finally, the extraction procedure is important for isolating active components from many organisms including plants [28] and fungi [29]. In this study, we used the ultrasonication method to isolate bioactive compounds from both fungal mycelia and the fruiting body. The results indicate that this method does extract compounds with antibacterial and anticancer activity. However, other parameters including the type of solvent should be considered, because different solvents have different polarities, and therefore extract compounds of different polarities. For example, the ethanolic mycelial extract showed more antibacterial and antibreast cancer cell line activity than those of the aqueous mycelial extract. These observations may be due to different bioactive compounds being extracted or different quantities of bioactive compound being extracted. Suitable extraction methods and extraction parameters should therefore be identified before large scale production of the bioactive compounds is attempted.

\section{CONCLUSION}

This study shows that extracts of the medicinal mushroom Sanghuangporus sp.1 exhibit significant in vitro antibacterial and anticancer activities. Furthermore, the extracts of laboratorygrown Sanghuangporus sp.1 mycelia are at least as active as those of the wild fruiting body of Sanghuangporus sp.1, and can therefore be used as a source of bioactive agents. Further studies are, however, required to isolate and identify the compounds responsible for the antibacterial and anticancer activities of Sanghuangporus sp.1.

\section{DECLARATIONS}

\section{Acknowledgement}

The authors are grateful to Mahasarakham University Faculty of Medicine for equipment support and language-editing assistance, and Mahasarakham University Faculty of Science for provision of laboratory space and equipment. We would also like to thank Ms. Jitdakan Naksuwankul, who collected mushroom sample and deposited it at Natural Medicinal Mushroom Museum, Mahasarakham University, Thailand. 


\section{Conflict of Interest}

No conflict of interest associated with this work.

\section{Contribution of Authors}

The authors declare that this work was done by the authors named in this article and all liabilities pertaining to claims relating to the content of this article will be borne by them.

\section{Open Access}

This is an Open Access article that uses a funding model which does not charge readers or their institutions for access and distributed under the terms of the Creative Commons Attribution License (http://creativecommons.org/licenses/by 14.0) and the Budapest Open Access Initiative (http://www.budapestopenaccessinitiative.org/rea d), which permit unrestricted use, distribution, and reproduction in any medium, provided the original work is properly credited.

\section{REFERENCES}

1. Sánchez C. Modem aspects of mushroom culture technology. Appl Microbiol Biotechnol 2004; 64: 756762.

2. Wasser SP. Medicinal mushroom as a source of antitumor and immunomodulating polysaccharides. Appl Microbiol Biotechnol 2002; 60: 258-274.

3. Moradali MF, Mostafavi H. Immunomodulating and anticancer agents in the realm of macromycetes fungi (macrofungi). Int Immunopharmacol 2007; 7: 701-724.

4. Hur JM, Yang CH, Han SH, Lee SH, You YO, Park JC, Kim KJ. Antibacterial effect of Phellinus linteus against methicillin-resistant Staphylococcus aureus. Fitoterapia 2004; 75: 603-605.

5. Kim SH, Song YS, Kim SK, Kim BC, Lim CJ, Park EH. Anti-inflammatory and related pharmacological activities of the $\mathrm{n}-\mathrm{BuOH}$ subfraction of mushroom Phellinus linteus. J Ethnopharmacol 2004; 93: 141-146.

6. Ayala-Zavala JF, Silva-Espinoza BA, Cruz-Valenzuela MR, Villegas-Ochoa MA, Esqueda M, González-Aguilar GA, Calderón-López Y. Antioxidant and antifungal potential of methanol extract of Phellinus spp. from Sonora, Mexico. Rev Iberoam Micol 2012; 29(3): 132138.

7. Huang GJ, Huang SS, Deng JS. Anti-inflammatory activities of inotilone from Phellinus linteus through the inhibition of MMP-9, NF-KB, and MAPK activation in vitro and in vivo. PLoS ONE 2012; 7(5): e35922.

8. Kang CM, Han DH, Hwang HK, Choi SH, Lee WJ. Anticancer effect of Phellinus linteus; potential clinical application in treating pancreatic ductal adenocarcinoma. J Carcinogene Mutagene 2013; Doi:10.4172/2157-2518.s9-001.
9. Li X, Wang J, Wang W, Liu C, Sun S, Gu J, Wang X, Boraschi D, Huang $Y, Q u D$. Immunomodulatory activity of a novel, synthetic beta-glucan ( $\beta$-glu6) in murine macrophages and human peripheral blood mononuclear cells. PLoS One 2013; 8(11): e80399.

10. Yan JK, Wang YY, Ma HL, Wang ZB, Pei JJ. Structural characteristics and antioxidant activity in vivo of a polysaccharide isolated from Phellinus linteus mycelia. $J$ Taiwan Inst Chem Eng 2016; 65: 110-117.

11. Park SK, Kim GY, Lim JY, Kwak JY, Bae YS, Lee JD, Oh YH, Ahn SC, Park YM. Acidic polysaccharides isolated from Phellinus linteus induce phenotypic and functional maturation of murine dendritic cells. Biochem Biophys Res Commun 2003; 312: 449-458.

12. Song $T Y$, Lin HC, Yang NC, Hu ML. Antiproliferative and antimetastatic effects of the ethanolic extract of Phellinus igniarius (Linnearus: Fries) Quelet. J Ethnopharmacol 2008; 115: 50-56.

13. Suabjakyong $P$, Saiki R, Van Griensven LJL, Higashi $K$, Nishimura K, Igarashi K, Toida T. Polyphenol extract from Phellinus igniarius protects against acrolein toxicity in vitro and provides protection in a mouse stroke model. PLOS ONE 2015; Doi:10.137/journal.pone. 0122733.

14. Chang HY, Ho YL, Sheu MJ, Lin YL, Tseng MC, Wu SH, Huang GJ, Chang YS. Antioxidant and free radical scavenging activities of Phellinus merrillii extracts. Bot Stud 2007; 48: 407-417.

15. Hwang BS, Lee IK, Choi HJ, Yun BS. Anti-influenza activities of polyphenols from the medicinal mushroom Phellinus baumii. Bioorg. Med Chem Lett 2015; 25 : 3256-3260.

16. Hong SB, Rhee $M H$, Yun BS, Lim YH, Song HG, Shin $K S$. Synergistic anti-bacterial effects of Phellinus baumii ethyl acetate extracts and $\beta$-lactam antimicrobial agents against methicillin-resistant Staphylococcus aureus. Ann Lab Med 2016; 36: 111-116.

17. Sittiwet $C$, Puangpronpitag $D$. Antibacterial activity of Phellinus gilvus aqueous extract. Int J Pharmacol 2008; 4(6): 500-502.

18. Sittiwet C, Puangpronpitag D. Anti-Staphylococcus aureus activity of Phellinus igniarius aqueous extract. Int J Pharmacol 2008; 4(6): 503-505.

19. Siriwattanametanon W, Kanchanarach W, Thiwthong R, Dodgson JLA. Culture filtrates from laboratory grown Phellinus mushrooms for use as antibacterial agents. Chiang Mai J Sci 2014; 41(1): 243-247.

20. Sangdee A, Sangdee K. Isolation, identification, culture and production of adenosine and cordycepin from cicada larva infected with entomopathogenic fungi in Thailand. Afr J Microbiol Res 2013; 7(2): 137-146.

21. Sangdee K, Nakbanpote W, Sangdee A. Isolation of the entomopathogenic fungal strain Cod-MK1201 from a cicada nymph and assessment of its antibacterial activities. Int Journal Med Mushrooms 2015; 17(1): 5163.

22. Buranrat B, Senggunprai L, Prawan A, Kukongviriyapan V. Simvastatin and atorvastatin as inhibitors of 
proliferation and inducers of apoptosis in human cholangiocarcinoma cells. Life Sci 2016; 153: 41-49.

23. Jung JY, Lee IK, Seok SJ, Lee HJ, Kim YH, Yun BS. Antioxidant polyphenols from the mycelial culture of the medicinal fungi Inonotus xeranticus and Phellinus linteus. J Appl Microbiol 2008; 104: 1824-1832.

24. Zaidman BZ, Tassin M, Mahajna J, Wasser SP. Medicinal mushroom modulators of molecular targets as cancer therapeutics. Appl Microbiol Biotechnol 2005; 67: 453-468.

25. Lee IK, Seok SJ, Kim WK, Yun BS. Hispidin derivatives from the Innonotus xeranticus and their antioxidan activity. J Nat Prod 2006; 69: 299-301.

26. Lee IK, Yun BS. Highly oxygenated and unsaturated metabolites providing a diversity of hispidin class antioxidants in the medicinal mushroom Inonotus and Phellinus. Bioorg Med Chem 2007; 15: 3309-3314.

27. Sun $Y$, Yin $T$, Chen $X H$, Zhang G, Curtis RB, Lu ZH, Jiang $\mathrm{JH}$. In vitro antitumor activity and structure characterization of ethanol extracts from wild and cultivated Chaga medicinal mushroom, Inonotus obliquus (Pers.:Fr.) Pilát (Aphyllphomycetideae). Int $J$ Med Mushrooms 2011; 13(2): 121-130.

28. Hsieh CW, Ko WC, Ho WJ, Chang CK, Chen GJ, Tsai JC. Antioxidant and hepatoprotective effects of Ajuga nipponensis extract by ultrasonic-assisted extraction. APJTM 2016; 9(5): 420-425.

29. Wang HJ, Pan MC, Chang CK, Chang SW, Hsieh CW. Optimization of ultrasonic-assisted extraction of cordycepin from Cordyceps militaris using orthogonal experimental design. Molecules 2014; 19: 20808-20820. 